\title{
Activité lipolytique de Penicillium chrysogenum
}

\author{
par \\ A. K. Chopra, Harish Chander, V. K. Batish \\ et B. Ranganathan *
}

\section{INTRODUCTION}

Les lipases microbiennes jouent un rôle important dans l'hydrolyse de la matière grasse, contribuant ainsi à la formation de flaveurs désirables ou indésirables dans les produits laitiers. Les champignons, on le sait, produisent des lipases [1, 2, 3, 4].

Dans notre travail précédent, les facteurs affectant la production de lipase par Penicillium chrysogenum ont été étudiés [5]. Le présent travail concerne la purification partielle de la lipase et ses propriétés enzymatiques.

\section{MATERIELS ET METHODES}

L'isolement et la production de lipase à partir de Penicillium chrysogenum ont été réalisés selon Chander et al. [5]. L'enzyme extracellulaire a ensuite été précipitée à l'aide de 40 à $60 \mathrm{p} .100$ d'une solution de sulfate d'ammonium à saturation.

L'activité lipasique a été déterminée par la méthode d'Oi et al. [6] avec quelques modifications. Le mélange réactif contenait $5 \mathrm{ml}$ d'émulsion à 5 p. 100 d'huile de beurre dans 7 p. 100 de gomme d'acacia, $5 \mathrm{ml}$ d'un tampon de 0,02 M Tris- $\mathrm{HCl}$ ( $\mathrm{pH} 7,5), 2 \mathrm{ml}$ d'une solution de $\mathrm{CaCl}_{2} / \mathrm{O}, 2 \mathrm{M}, 1 \mathrm{ml}$ de solution enzymatique et $2 \mathrm{ml}$ d'eau distillée. La protéine fut testée par la méthode de Lowry [7].

Unité de lipase $=$ la quantité de soude $\mathrm{N} / 100$ nécessaire pour neutraliser les acides libérés du substrat par $1 \mathrm{ml}$ de l'enzyme à $37^{\circ} \mathrm{C}$ en $3 \mathrm{~h}$.

\footnotetext{
* Division of Dairy Bacteriology, National Dairy Research Institute, Karnal-132001, India.
} 

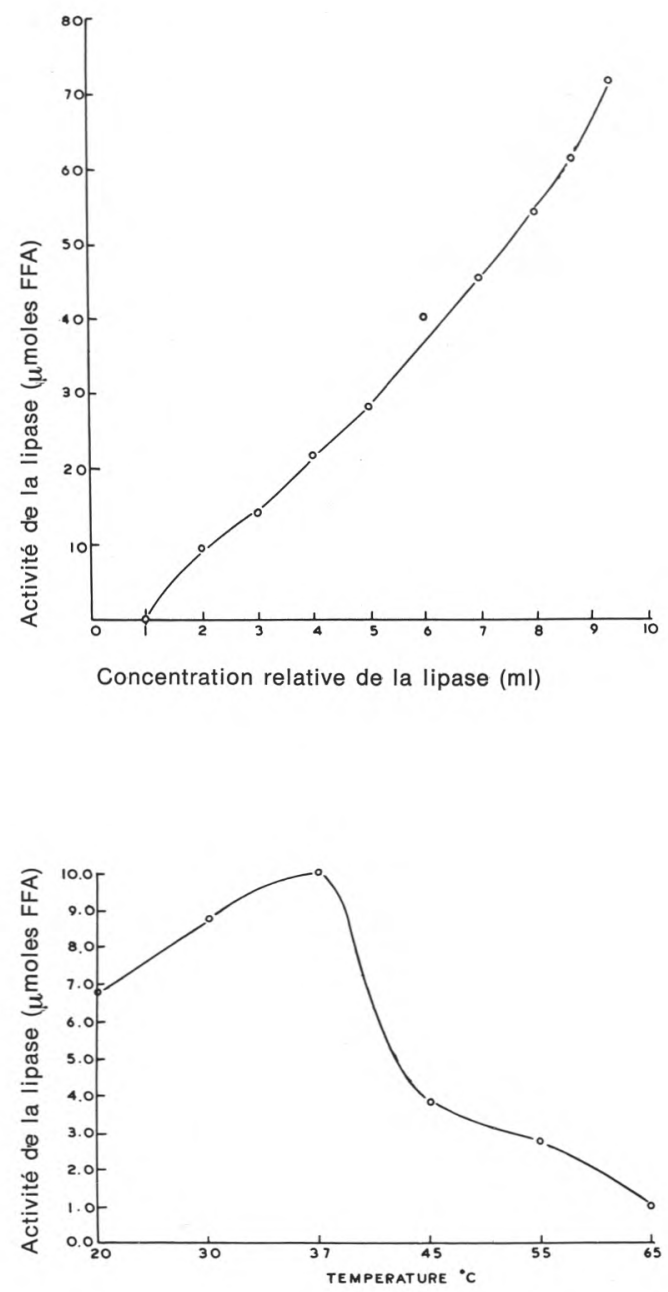

fig. 1

Effet des concentrations de l'enzyme sur l'activité de la lipase de $P$. chrysogenum.

fig. 2

Effet de la température sur l'activité de la lipase de $P$. chrysogenum.

\section{RESULTATS}

Sur la base protéique, cette fraction partiellement purifiée avait 12 fois plus d'activité que l'enzyme brute.

Si la concentration de l'enzyme (ml) croissait, l'activité lipasique augmentait dans la même proportion (fig. 1).

L'activité lipasique maximale ( 9,6 unités) fut observée à $37^{\circ} \mathrm{C}$ pour Penicillium chrysogenum. Au-delà de cette température du mélange réactif, cette activité diminuait (fig. 2). 

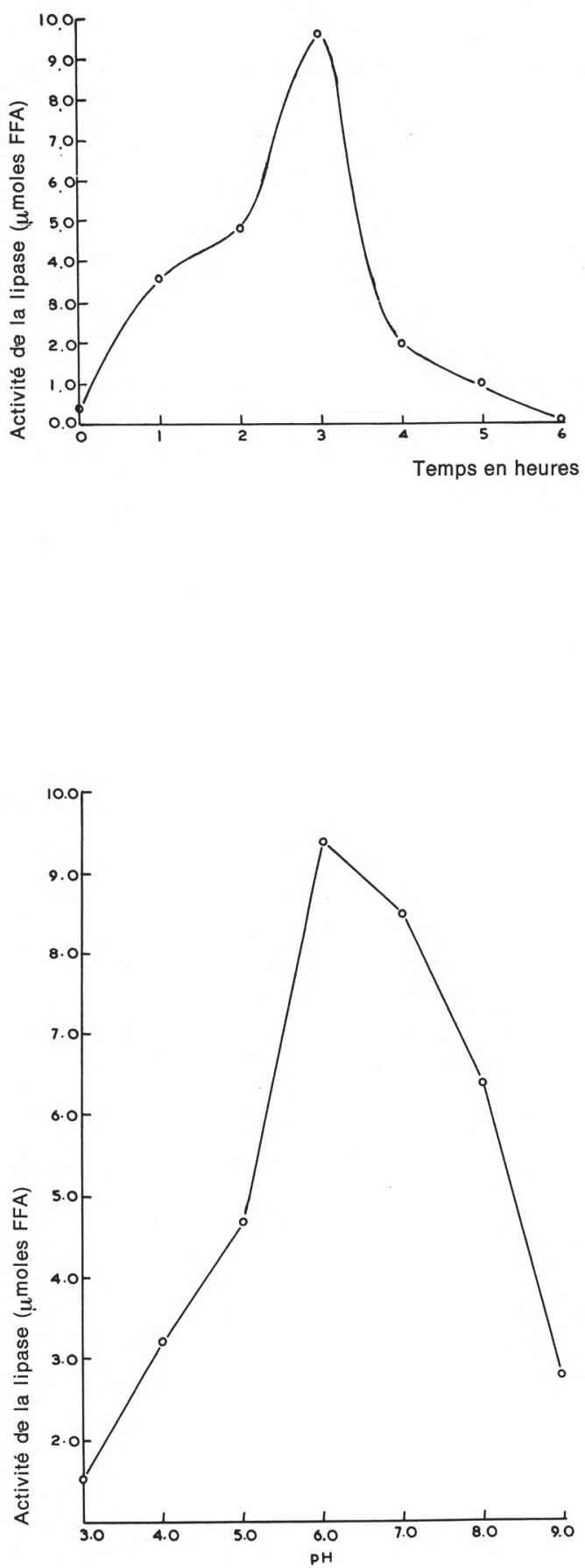

fig. 3

Effet du temps d'incubation du mélange réactif sur l'activité de la lipase de $P$. chrysogenum.

fig. 4

Effet du pH sur l'activité de la lipase de $P$. Chrysogenum. 
L'activité maximale était obtenue après une incubation de $3 \mathrm{~h}$ à $37^{\circ} \mathrm{C}$ (fig. 3).

Le $\mathrm{pH}$ optimum pour la production de lipase à partir de Penicillium chrysogenum fut noté à 6 . Un retard considérable d'activité lipasique est causé lorsque le $\mathrm{pH}$ s'élève à 9 ou s'abaisse à 3 (fig. 4).

Quant aux substrats lipidiques utilisés pour apprécier l'activité de la lipase, ils sont présentés dans le tableau 1 . Le degré d'hydrolyse

\section{TABLEAU 1}

Spécificité du substrat envers la lipase de Penicillium chrysogenum

\begin{tabular}{l|c|c}
\hline \multicolumn{1}{c|}{ Source lipidique } & $\begin{array}{c}\text { Activité de la lipase } \\
\text { (unités) }\end{array}$ & $\begin{array}{c}\text { Activité relative } \\
\text { (p. 100) }\end{array}$ \\
\cline { 2 - 3 } & & 100 \\
Tributyrine & 11,2 & 81 \\
Tricapröne & 9,2 & 60 \\
Tripropionine & 6,7 & 42 \\
Tricapryline & 4,7 & 45 \\
Huile de beurre & 5,1 & 34 \\
Huile d'olive & 3,8 & 20 \\
Huile de noix de coco & 2,2 & 18 \\
Huile de moutarde & 2,0 & 10 \\
Huile de graine de coton & 1,1 & \\
& & \\
\hline
\end{tabular}

des triglycérides synthétiques par la lipase partiellement purifiée est, par ordre décroissant, le suivant : tributyrine, tricaproïne, tripropionine, tricapryline, avec les activités relatives : $100,81,60$ et 42 p. 100 respectivement.

Pour les triglycérides naturels : huile de beurre, huile d'olive, huile de coco, huile de moutarde, huile de graine de coton, avec les activités relatives : $45,34,20,18$ et 10 p. 100 respectivement. (fig. 5).

L'enzyme était complétement inactivée à $70^{\circ} \mathrm{C}$ pendant $10 \mathrm{~min}$

L'activité enzymatique était stimulée par $\mathrm{Mg}++, \mathrm{Mn}++, \mathrm{Fe}++$, $\mathrm{Na}+$ et $\mathrm{Ca}++$ jusqu'à $32,22,7,7$ et 23 p. 100 respectivement, et inhibée par $\mathrm{Zn}++, \mathrm{Co}++, \mathrm{Fe}+++, \mathrm{Li}+++$ et $\mathrm{Cu}++$, jusqu'à, respectivement, 20, 22, 17, 45 et 46 p. 100 (tab. 2).

Le tableau 3 montre les proportions de l'accroissement de l'activité lipasique ou son inhibition par d'autres éléments. 


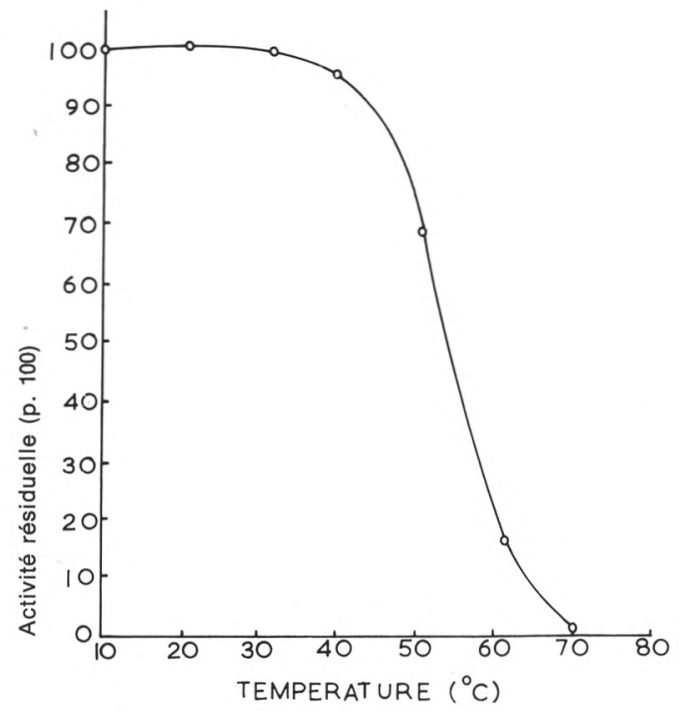

fig. 5

Effet du traitement thermique sur la stabilité de la lipase de $P$. chrysogenum.

TABLEAU 2

Effet de sels métalliques sur l'activité de la lipase de Penicillium chrysogenum

\begin{tabular}{|c|c|c|c|c|}
\hline $\begin{array}{l}\text { Sels } \\
\text { métalliques }\end{array}$ & Concentration & $\begin{array}{l}\text { Activité } \\
\text { lipasique } \\
\text { (unités) }\end{array}$ & $\begin{array}{l}\text { Activité } \\
\text { relative }\end{array}$ & $\begin{array}{c}\text { Action } \\
(+ \text { ou }-) \\
\text { p. } 100\end{array}$ \\
\hline $\begin{array}{l}\text { Témoin } \\
\mathrm{CuSo}_{4} \\
\mathrm{FeSo}_{4} \\
\mathrm{FeCl}_{3} \\
\mathrm{LiCl}_{3} \\
\mathrm{ZnSo}_{4} \\
\mathrm{CaCl}_{2} \\
\mathrm{MgCl}_{2} \\
\mathrm{MnCl}_{2} \\
\mathrm{NaCl}_{2} \\
\mathrm{CaCl}_{2}\end{array}$ & $\begin{array}{c}\overline{-} \\
10-2 \mathrm{M} \\
" \\
" \\
" \\
" \\
" \\
" \\
" \\
" \\
"\end{array}$ & $\begin{array}{r}9,4 \\
5,1 \\
10,0 \\
8,8 \\
5,2 \\
7,5 \\
7,3 \\
12,4 \\
11,5 \\
10,0 \\
11,6\end{array}$ & $\begin{array}{r}100 \\
54 \\
107 \\
83 \\
55 \\
80 \\
78 \\
132 \\
122 \\
107 \\
123\end{array}$ & $\begin{array}{l}- \\
-46 \\
+\quad 7 \\
-17 \\
-45 \\
-20 \\
-22 \\
+32 \\
+22 \\
+7 \\
+23\end{array}$ \\
\hline
\end{tabular}




\section{TABLEAU 3}

Effet d'inhibiteurs sur l'activité de la lipase de Penicillium chrysogenum

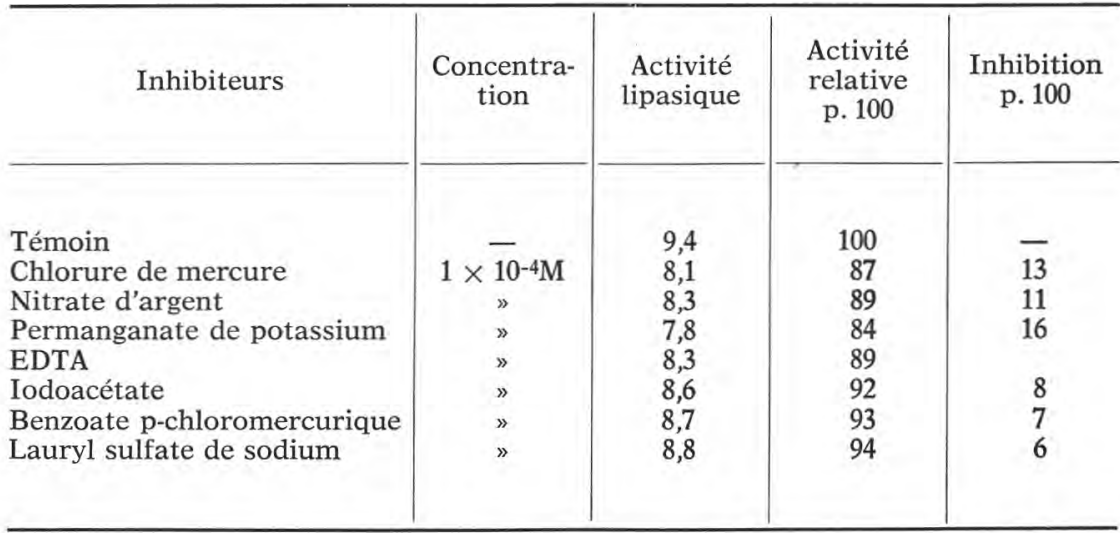

\section{DISCUSSION}

Les résultats sur la relation linéaire entre l'activité et la concentration enzymatique de la lipase de Penicillium chrysogenum sont semblables à ceux observés avec la lipase purifiée de Propionibacterium shermanii [8] et de Pseudomonas aeruginosa [9].

L'activité maximale observée à $37^{\circ} \mathrm{C}$ avec Penicillium chrysogenum s'est révélée en accord partiel avec les observations concernant Penicillium roqueforti, Geotrichum candidum et Eremothecium ashbii $[10,11,12]$.

Au contraire, une température optimale plus élevée a été également observée pour l'activité de lipases microbiennes [11, 13].

Les lipases produites par Aspergillus niger et Rhizopus delemar ont le même $\mathrm{pH}$ optimum d'activité 6,0 que $P$. chrysogenum $[14,15]$ alors que Eitenmiller et al. [10] ainsi que Oi et al. ont rapporté que le $\mathrm{pH}$ optimum était de 8,0 pour $P$. roqueforti et $P$. crustosum.

$P$. chrisogenum hydrolyse la tributyrine par préférence aux autres acides gras. Shipe [16], Morris et Jereski [1], Eitenmiller et al. [10] ont observé le même fait pour la lipase de $P$. roqueforti. La spécificité de la lipase pour les acides gras à chaîne courte dans les triglycérides simples peut être attribuée aux différences de poids moléculaires et à l'état physique des différents substrats.

Nos résultats sur la stabilité à la chaleur ne sont pas corroborés par le résultat des travaux de Eitenmiller et al. [17] dans le cas de $P$. roqueforti, car ces auteurs ont noté l'inhibition de l'activité de sa lipase pour une température de $50^{\circ} \mathrm{C}$ pendant $10 \mathrm{~min}$. Cependant, 
Iwai et al. [18] ont observé que les lipases purifiées B et C de Rhizopus delemar étaient stables au-dessous de $45^{\circ} \mathrm{C}$ tandis que la lipase A l'était au-dessous de $60^{\circ} \mathrm{C}$.

La lipase obtenue de $P$. chrysogenum a été stimulée par $\mathrm{Ca}++$, $\mathrm{Mg}++, \mathrm{Mn}++$ et $\mathrm{Na}+$. Les résultats ci-dessus donnés concordent avec les observations de Eitenmiller et al. [10] qui ont rapporté que l'activité de la lipase de $P$, roqueforti était accrue par $\mathrm{Mg}++$ et $\mathrm{Mn}++$. Le mécanisme de l'action des ions calcium sur l'activité lipasique a été expliqué par Ota et Yamada [19] qui ont remarqué que les ions calcium provoquaient l'étalement de l'enzyme sur la molécule du substrat. Ota et al. [20] ont également trouvé une stimulation de l'activité de la lipase de Candida paralipolytica par les ions sodium en l'attribuant à la force ionique élevée des sels de sodium.

Les résultats des recherches sur l'effet inhibiteur du sel de cuivre sur l'activité de la lipase de $P$. chrysogenum sont semblables à ceux rapportés à propos de la lipase produite par des micro-organismes appartenant aux espèces telles que Torulopsis [21] et Rhizopus [22]. L'inhibition de la lipase de $P$. chrysogenum par le sel de zinc est comparable à celle concernant d'autres lipases microbiennes, Aspergillus niger [23], P. roqueforti [10] et L. brevis [24].

Quelques agents spécifiques $\mathrm{SH}$, tels que le benzoate p-chloromercurique, l'iodoacétate et le chlorure mercurique exercent une légère action inhibitrice sur l'activité de la lipase de $P$. chrysogenum. Ces résultats sont comparables à ceux fournis par Somkuti et al. [25] et Oterholm et al. [8] concernant Mucor pusillus et Propionibacterium shermanii. Une diminution d'activité de la lipase de $P$. chrysogenum par le permanganate de potasse mentionné dans la présente étude est comparable à celle signalée pour la lipase de Mucor par Nagaoka et Yamada [2].

\section{Rés u mé}

La lipase extracellulaire de Penicillium chrysogenum a été purifiée partiellement au douzième à l'aide de 40 à $60 \mathrm{p}$. 100 d'une solution de sulfate d'ammonium à saturation. Cette lipase hydrolyse les triglycérides simples, à savoir tributyrine, tricaproïne, tripropionine et tricapryline, en ordre décroissant, et l'huile de beurre plus aisément que l'huile de noix de coco. L'activité maximale a été observée au $\mathrm{pH} 6,0$ et à $37^{\circ} \mathrm{C}$ après $3 \mathrm{~h}$ d'incubation. Les sels de calcium, sodium, ferreux, manganèse et magnésium ont une action stimulante, tandis que les sels de cuivre, cobalt, lithium, ferrique et zinc sont inhibiteurs, ainsi que le chlorure mercurique, le nitrate d'argent, le permanganate de potassium, l'iodoacétate, le benzoate p-chloromercurique et le laurylsulfate de sodium. Le permanganate de potassium a été trouvé exercer l'action inhibitrice la plus forte. Par ailleurs, l'enzyme est totalement inactivée en $10 \mathrm{~min}$ à $70^{\circ} \mathrm{C}$. 


\section{S u $\mathrm{m} \mathbf{m}$ a r y}

\section{FACTORS AFFECTING LIPASE PRODUCTION FROM PENICILLIUM CHRYSOGENUM}

Factors affecting growth and lipase production by Penicillium chrysogenum were studied. The optimum conditions for lipase production by the mold were $30^{\circ} \mathrm{C}$ at $\mathrm{pH}$ 6.0. In shake cultures, lipase production was enhanced by 70 p. 100 . Amongst several carbohydrates that were examined, maximum lipase production occurred in medium containing glucose, followed by maltose, mannitol, galactose, sucrose, lactose and fructose in decreasing order. Out of different nitrogen sources, peptone proved most satisfactory.

Reçu pour publication en novembre 1979.

\section{Bibliographie}

[1] Morris (H. A.), Jezeski (J. J.) (1953). - J. Dairy Sci., 36, 1285.

[2] Nagaoka (K.), Yamada (Y.) (1973). - Agr. Biol. Chem., 37, 2791.

[3] Benzonana (G.) (1974). - Lipids, 9, 166.

[4] Kinsella (J. E.), Hwang (D.) (1976). - Biotech. Bioeng., 18, 927.

[5] Chander (H.), Sannabhadti (S. S.), Elias (J.), Ranganathan (B.) (1977). J. Fd. Sci., 42, 1482.

[6] Oi (S.), Swada (A.), Satomura (Y.) (1967). - Agr. Biol. Chem., 31, 1357.

[7] Lowry (O. H.), Rosebrough (H. J.), FarR (A. L.), Randall (R. J.) (1951). J. Biol. Chem., 193, 265.

[8] Oterholm (A.), Ordal (Z. J.), Witter (L. D.) (1970), - Appl. Microbiol., 20, 16.

[9] NADKARNI (S. R.) (1971). - Enzymologica, 40, 302.

[10] Eitenmiller (R. R.), Vakil (J. R.), Shahani (K. M.) (1970). - J. Fd. Sci., $35,130$.

[11] Tsumisaka (Y.), Iwai (M.), Tominaga (Y.) (1973). - Agr. Biol. Chem., 37, 1457.

[12] Shen (Y. C.), LiU (T. C.), Chang (C. L.), Wang (L. W.) (1975). - Microbiol. Abstr., 10, 10 A, 2990.

[13] Liu (W. H.), Beppu (T.), Arima (K.) (1973). - Agr. Biol. Chem., 37, 2493.

[14] Fukumoto (J.), Iwai (M.), TsujisaKa (Y.) (1963). - J. Gen. Appl, Microbiol., 9. 353.

[15] Fukumoto (J.), Iwai (M.), TsujisaKa (Y.) (1964). - J. Gen. Appl. Microbiol., $10,257$.

[16] SHIPE (W. F.) (1951). - Arch. Biochem. Biophys., 30, 165.

[17] Eitenmiller (R. R.), Vakil (J.R.), Shahant (K. M.) (1968). - J. Dairy Sci., 51, 940.

[18] Iwai (M.), Tsujisaka (Y.) (1974). - Agr. Biol. Chem., 38, 1241.

[19] Ota (Y.) Yamada (K.) (1967). - Agr. Biol. Chem., 31, 809.

[20] Ota (Y), Teruaki (N.), Yamada (K.) (1972). - Agr. Biol. Chem., 36, 1895.

[21] Motai (H.), Ichishima (E.), Yoshida (F.) (1966). - Nature, 210, 308.

[22] OI (S.), Yamazaski (O.), Sawada (A.), Satomura (Y.) (1969). - Agr. Biol. Chem., 33, 729.

[23] Iwai (M.), Tsujisaka (Y.), Fukumoto (J.) (1964). - J. Gen. Appl. Microbiol., $10,87$.

[24] Chander (H.), Chebbi (N. B.), Ranganathan (B.) (1973). - Arch. Fur Microbiol., 92, 171.

[25] Somkuti (G. A.), Babel (F. J.), Somkuti (A. C.) (1969). - Appl. Microbiol., 17,606 . 